TRANSACTIONS OF THE

AMERICAN MATHEMATICAL SOCIETY

Volume 363, Number 6, June 2011, Pages 3277-3285

S 0002-9947(2011)05231-1

Article electronically published on January 14, 2011

\title{
SHORT PRESENTATIONS FOR ALTERNATING AND SYMMETRIC GROUPS
}

\author{
J. N. BRAY, M. D. E. CONDER, C. R. LEEDHAM-GREEN, AND E. A. O'BRIEN
}

\begin{abstract}
We construct two kinds of presentations for the alternating and symmetric groups of degree $n$ : the first are on two generators in which the number of relations is $O(\log n)$ and the presentation length is $O\left(\log ^{2} n\right)$; the second have a bounded number of generators and relations and length $O(\log n)$.
\end{abstract}

\section{INTRODUCTION}

A long-standing question is the existence of 'simple' presentations for the alternating and symmetric groups of degree $n$. Many such presentations were recorded by Coxeter and Moser in their seminal monograph [8, $\S \S 6.2-6.3]$, and while the term 'simple' was not well defined, the number of generators and relators certainly influenced their selections.

A question of more recent interest, motivated in part by algorithmic group theory, is the existence of 'short' presentations. In a 1984 paper, Babai and Szemerédi [3] defined the length of a presentation to be the number of symbols required to write down the presentation. Each generator is a single symbol, and a relator is a string of symbols, where exponents are written in binary. The length of a presentation is the number of generators plus the sum of the lengths of the relators. This definition accords well with the requirement of polynomial-time analysis of computational complexity.

In [3, Babai and Szemerédi formulated the Short Presentation Conjecture: there exists a constant $c$ such that every finite simple group $G$ has a presentation of length $O\left(\log ^{c}|G|\right)$. One motivation for this conjecture is its application to deciding in polynomial time certain key properties of finite matrix groups, such as order and membership. We refer the reader to the survey by Babai [4 for a discussion on these and related matters. The results of Babai et al. 1], Hulpke \& Seress [12, and Suzuki [20] establish this conjecture with $c=2$ for all finite simple groups, with the possible exception of the Ree groups ${ }^{2} G_{2}(q)$.

Perhaps the best known family of presentations for the finite symmetric groups are the presentations of Moore [14; see also 8, 6.22]. In these, the symmetric group $S_{n}$ of degree $n$ is presented in terms of the transpositions $t_{k}=(k, k+1)$ for

Received by the editors September 21, 2009 and, in revised form, October 1, 2009.

2000 Mathematics Subject Classification. Primary 20F05, 20 B30.

This work was supported in part by the Marsden Fund of New Zealand via grant UOA 0412. We thank Bob Guralnick and Bill Kantor for discussions on this topic in 2005. We thank the referee for detailed commentary, and George Havas and Igor Pak for their feedback. We are particularly grateful to Bill Kantor for his extensive and most helpful suggestions on drafts of this paper.

(c)2011 American Mathematical Society Reverts to public domain 28 years from publication 
$1 \leqslant k<n$, which generate $S_{n}$ and satisfy the defining relations $t_{k}^{2}=1$ for $1 \leqslant k<n$, and $\left(t_{k-1} t_{k}\right)^{3}=1$ for $1<k<n$, and $\left(t_{j} t_{k}\right)^{2}=1$ for $1 \leqslant j<k-1<n-1$. These are now commonly known as Coxeter relations for $S_{n}$, because of their analogues in other contexts. For $n>1$ the number of these relations is $n(n-1) / 2$, and since each relator has bounded length, the presentation length is $O\left(n^{2}\right)$. By using a single transposition and an $n$-cycle as generators, Moore derived a shorter presentation of length $O(n \log n)$. By introducing powers of the $n$-cycle as (redundant) additional generators, one can readily obtain a presentation for $S_{n}$ with $\lfloor n / 2\rfloor+1$ generators, $2\lfloor n / 2\rfloor+2$ relations, and length $O(n)$.

We now describe the main results of this paper. In Section 2 we introduce methods of 'gluing' together presentations for $S_{m}$ and $S_{n}$ to produce presentations for $S_{m+n-1}$ and $S_{m+n}$, which are then used to prove the following.

Theorem 1.1. For every integer $n>1$, the symmetric group $S_{n}$ has a presentation on the generators $(1,2)$ and $(1,2, \ldots, n)$ in which the number of relations is $O(\log n)$ and the presentation length is $O\left(\log ^{2} n\right)$.

In Section 3 we adapt these methods to construct presentations for $S_{n}$ with a uniformly bounded number of generators and relations and length $O(\log n)$. Note that $O(\log n)$ is the best possible presentation length, since merely specifying the integer $n$ requires $\Omega(\log n)$ bits. We use presentations for 2-dimensional projective linear groups to produce presentations for $S_{p+q+2}$ for odd primes $p$ and $q$, and then apply (proven) variants of the Goldbach conjecture.

Before proceeding, we define some notation in order to ease exposition. If $P=$ $\{X \mid \mathcal{R}\}$ is a presentation, then $d(P)$ and $r(P)$ denote the cardinalities of $X$ and $\mathcal{R}$, respectively, and $\ell(P)$ is the length of $P$. The standard generators for $S_{n}$ are the transposition $(1,2)$ and the $n$-cycle $(1,2, \ldots, n)$. Note that $(1,2)$ can be replaced by any transposition $(k, k+1)$ for $1 \leqslant k<n$, and that the choice of $k$ is irrelevant, since conjugation by $(1,2, \ldots, n)$ is equivalent to the obvious cyclic relabelling of the points. A presentation for $S_{n}$ whose generating set contains the standard generators is called special. Special presentations can be particularly helpful in the constructive recognition of linear groups.

Our main theorem in Section 3 is the following.

Theorem 1.2. For every integer $n>1$, the symmetric group $S_{n}$ has a special presentation $P$ of length $O(\log n)$ with a uniformly bounded number of generators and relations. In particular, if $n$ is even and $n-2$ is the sum of two odd primes, then $d(P) \leqslant 6$ and $r(P) \leqslant 24$, while if $n$ is odd and $n-3$ is the sum of two odd primes, then $d(P) \leqslant 7$ and $r(P) \leqslant 28$; for all other values of $n, d(P) \leqslant 15$ and $r(P) \leqslant 82$.

We are left with one outstanding question: Does $S_{n}$ have a bounded presentation of length $O(\log n)$ on its standard generators?

By a theorem of Babai, Kantor and Lubotzky 2], the diameter of the Cayley graph for $S_{n}$ on the standard generators is $\Theta\left(n^{2}\right)$. Hence if each nonstandard generator in a special presentation is replaced by a word in the standard generators, then the length of every relator is multiplied by at most $n^{2}$. Theorem 1.2 now implies that $S_{n}$ has a bounded presentation of length $O\left(n^{2} \log n\right)$ on its standard generators. By exploiting the ideas used in the proof of Theorem 1.2 and a short presentation for $\operatorname{PGL}(2, p)$ given in [8, §7.5], we can reduce the length to $O\left(n^{2}\right)$. Whether or not there is a shorter bounded presentation is an open question. 
Our presentations for $S_{n}$ give rise to presentations for the alternating group $A_{n}$ with similar characteristics, as we explain in Section 4. In particular, we have the following consequence of Theorems 1.1 and 1.2

Theorem 1.3. For every integer $n>2$, the alternating group $A_{n}$ has:

(a) a 2-generator presentation with $O(\log n)$ relations and length $O\left(\log ^{2} n\right)$;

(b) a presentation of length $O(\log n)$ with a uniformly bounded number of generators and relations;

(c) a 2-generator presentation with a uniformly bounded number of relations.

Theorem 1.3 (c) provides a positive answer to a question of Campbell et al. 7 . about the existence of a 2-generator presentation for $A_{n}$ with a bounded number of relators.

A weaker version of some of these results was announced in 2004; see [15], where our original motivation is also described - namely the challenge of finding short presentations for groups of Lie type on specific generating sets. In particular, our presentations for $A_{n}$ and $S_{n}$ can be used in conjunction with the algorithms of Beals et al. [5] to verify constructive recognition of black-box representations of these groups.

Simultaneously, and independent of our work, Guralnick, Kantor, Kassabov and Lubotzky obtained similar but stronger results about short presentations of $A_{n}$ and $S_{n}$ with a bounded number of generators and relations.

In [10], Guralnick et al. use a more refined definition of presentation length, namely the number of generators plus the sum of the lengths of the relators as words in the corresponding free group (see [18, pp. 190-191]), and obtain a result which is optimal for that metric. They also exploit properties of $\operatorname{PSL}(2, p)$, but in other respects their approach is substantially simpler; for example, instead of the Goldbach conjecture, they use Bertrand's Postulate (proved by Chebyshev in 1850).

In a major extension, they use their presentations for $A_{n}$ and $S_{n}$ to prove that every nonabelian finite simple group of rank $r$ over $\operatorname{GF}(q)$, with the possible exception of the Ree groups ${ }^{2} G_{2}(q)$, has a presentation with a bounded number of generators and relations and total length $O(\log r+\log q)$; again this result is optimal. In a more recent paper [11, they show that both $A_{n}$ and $S_{n}$ have presentations with 3 generators, 7 relators, and length $O(\log n)$.

After we completed this work we learned from Bill Kantor about a 1972 construction by Sass [17] of a short presentation for $A_{p+2}$ for prime $p$. This could be used to obtain similar results.

\section{Building PRESEntations FOR $S_{m+n}$ FROM $S_{m}$ AND $S_{n}$}

We first introduce simple constructions to build special presentations for $S_{m+n-1}$ and $S_{m+n}$ from special presentations for $S_{m}$ and $S_{n}$. The key idea is to 'glue' the presentations together using a single transposition.

Lemma 2.1. Given special presentations $P_{m}$ and $P_{n}$ for the symmetric groups $S_{m}$ and $S_{n}$ of degrees $m, n \geqslant 3$, there exist special presentations $P_{m+n-1}$ and $P_{m+n}$ for $S_{m+n-1}$ and $S_{m+n}$, respectively, such that:

$$
\begin{array}{ll}
d\left(P_{m+n-1}\right) \leqslant d\left(P_{m}\right)+d\left(P_{n}\right)+1, & d\left(P_{m+n}\right) \leqslant d\left(P_{m}\right)+d\left(P_{n}\right)+2, \\
r\left(P_{m+n-1}\right) \leqslant r\left(P_{m}\right)+r\left(P_{n}\right)+8, & r\left(P_{m+n}\right) \leqslant r\left(P_{m}\right)+r\left(P_{n}\right)+12, \\
\ell\left(P_{m+n-1}\right) \leqslant \ell\left(P_{m}\right)+\ell\left(P_{n}\right)+O(1), & \ell\left(P_{m+n}\right) \leqslant \ell\left(P_{m}\right)+\ell\left(P_{n}\right)+O(1) .
\end{array}
$$


Moreover, if $d\left(P_{m}\right)=d\left(P_{n}\right)=2$, then in each case the resulting presentation can be simplified to one on the two standard generators, with at most $r\left(P_{m}\right)+r\left(P_{n}\right)+12$ relations, and length $\ell\left(P_{m}\right)+\ell\left(P_{n}\right)+O(\log m+\log n)$.

Proof. Let $P_{m}=\{A \mid \mathcal{R}\}$ and $P_{n}=\{B \mid \mathcal{S}\}$, with $(a, v)$ and $(b, w)$ standing for the standard generators of $S_{m}$ and $S_{n}$. We first show that

$$
\begin{gathered}
P_{m+n}=\left\{A, B, t, y \mid \mathcal{R}, \mathcal{S}, t^{2},(a t)^{3},(t b)^{3},[a, b],[a, w],[v, b],[v, w],\right. \\
\left.[a v, t],\left[v^{-1}, t\right],[t, w b],\left[t, w^{-1} b w\right], y^{-1} w t v\right\}
\end{gathered}
$$

is a special presentation for $S_{m+n}$.

Let $G$ be the group defined by this presentation, and observe that there exists an epimorphism $\theta: G \rightarrow S_{m+n}$ under which

$$
\begin{aligned}
a & \mapsto(m-1, m), & v & \mapsto(1,2, \ldots, m), \\
b & \mapsto(m+1, m+2), & w & \mapsto(m+1, m+2, \ldots, m+n), \\
t & \mapsto(m, m+1), & y & \mapsto(1,2, \ldots, m, m+1, m+2, \ldots, m+n) .
\end{aligned}
$$

Now in $G$, define $t_{m-1}=a$ and $t_{m-i-1}=v^{i} a v^{-i}$ for $1 \leqslant i \leqslant m-2$, and also $t_{m}=t$, and $t_{m+1}=b$ and $t_{m+i+1}=w^{-i} b w^{i}$ for $1 \leqslant i \leqslant n-2$.

By the choice of $a$ and $v$, the elements $t_{i}$ for $1 \leqslant i<m$ generate a subgroup isomorphic to $S_{m}$ and hence satisfy the Coxeter relations for $S_{m}$, and similarly, the elements $t_{m+i}$ for $1 \leqslant i<n$ satisfy the Coxeter relations for $S_{n}$. Also the relations $(a t)^{3}=(t b)^{3}=1$ imply that $\left(t_{i} t_{i+1}\right)^{3}=1$ for $1 \leqslant i \leqslant m+n-2$.

Next, we show that $\left[t_{j}, t_{k}\right]=\left(t_{j} t_{k}\right)^{2}=1$ whenever $1 \leqslant j<k-1<m+n-1$. If $j<k<m$ or $m<j<k$, then this follows from the presentation $P_{m}$ or $P_{n}$, respectively; if $j<m<k$, then it follows from the four relations $[a, b]=[a, w]=$ $[v, b]=[v, w]=1$, as these imply that $\langle a, v\rangle$ commutes with $\langle b, w\rangle$. The elements $a v$ and $v a v^{-1}$ generate a subgroup of index $m$ in $\langle a, v\rangle \cong S_{m}$ containing the involutions $t_{1}, t_{2}, \ldots, t_{m-2}$, and $w b$ and $w^{-1} b w$ generate a subgroup of index $n$ in $\langle b, w\rangle \cong$ $S_{n}$ containing $t_{m+2}, t_{m+3}, \ldots, t_{m+n-1}$. Accordingly, the four relations $[a v, t]=$ $\left[v a v^{-1}, t\right]=[t, w b]=\left[t, w^{-1} b w\right]=1$ imply that $t$ centralises $\left\langle t_{1}, t_{2}, \ldots, t_{m-2}\right\rangle$ and $\left\langle t_{m+2}, t_{m+3}, \ldots, t_{m+n-1}\right\rangle$, and so we obtain also $\left[t_{j}, t_{m}\right]=1$ for $1 \leqslant j \leqslant m-2$ and $\left[t_{m}, t_{k}\right]=1$ for $m+2 \leqslant k \leqslant m+n-1$.

Hence the $m+n-1$ involutions $t_{i}$ generate a subgroup satisfying the Coxeter relations for $S_{m+n}$. The relations in $P_{m}$ and $P_{n}$ imply that each of the elements of $A$ or $B$ is expressible as a word in these $t_{i}$, and the relation $y^{-1} w t v=1$ then implies the same for $y$, and therefore the involutions $t_{i}$ generate $G$. Thus $G$ is isomorphic to $S_{m+n}$, and the rest follows easily, by observing that $a=y t y^{-1}$, $v=(y t)^{m-1} y^{-(m-1)}, \quad b=y^{-1} t y$ and $w=y^{-(n-1)}(t y)^{n-1}$.

A similar construction without the gluing transposition gives the following special presentation for $S_{m+n-1}$ with the required properties:

$$
\begin{array}{r}
P_{m+n-1}=\left\{A, B, y \mid \mathcal{R}, \mathcal{S},(a b)^{3},[a, w b],\left[a, w^{-1} b w\right],[v, w b],\left[v, w^{-1} b w\right],\right. \\
\left.[a v, b],\left[v a v^{-1}, b\right], y^{-1} w v\right\} .
\end{array}
$$

As a basis for induction, the constructions used in Lemma 2.1 are not the best possible, since doubling the degree roughly doubles both the number of relations and the presentation length. Taking $m=n$, however, we can express one copy of $S_{n}$ as a conjugate of another and obtain the following.

Lemma 2.2. Given a special presentation $P_{n}$ for $S_{n}$ where $n \geqslant 3$, there exists a special presentation $Q_{2 n}$ for $S_{2 n}$ with $d\left(Q_{2 n}\right) \leqslant d\left(P_{n}\right)+1, \quad r\left(Q_{2 n}\right) \leqslant r\left(P_{n}\right)+6$, 
and $\ell\left(Q_{2 n}\right) \leqslant \ell\left(P_{n}\right)+O(1)$. Moreover, if $d\left(P_{n}\right)=2$, then $Q_{2 n}$ can be simplified to one on the two standard generators, with at most $r\left(P_{n}\right)+6$ relations, and length $\ell\left(P_{n}\right)+O(\log n)$.

Proof. As in the proof of Lemma 2.1 one can show that if $x, w$ stand for the standard generators of $S_{n}$ in $P_{n}$, then

$$
\left\{A, y \mid \mathcal{R}, y^{2 n},(x y)^{2 n-1},\left[x, w y^{-1}\right],\left[w^{2} x w^{-1}, w y^{-1}\right],\left[x, y^{n}\right]^{2},\left[x, y^{n-1}\right]^{2}\right\}
$$

is a special presentation for $S_{2 n}$, with $y$ standing for the $2 n$-cycle $(1,2, \ldots, 2 n)$. In particular, $w=y^{-(n-1)}(y x)^{n-1}$ (since this relation is satisfied by the corresponding elements in $S_{2 n}$ ), and the final assertion follows.

Theorem 1.1 now follows from Lemma 2.2 and its obvious analogue for $S_{2 n-1}$.

\section{Short PRESEntations For $S_{n}$ With A BOUNDED NUMBER OF RELATIONS}

As a first step in this section, we give a variant of our 'gluing' constructions.

Lemma 3.1. Let $P=\{A, t \mid \mathcal{R}\}$ and $Q=\{B, t \mid \mathcal{S}\}$ be presentations for $S_{m+1}$ and $S_{n+1}$, with $m, n \geqslant 1$, such that the generator $t$ stands for a transposition in both cases. Let $M$ and $N$ be sets of words on the sets $A \cup\{t\}$ and $B \cup\{t\}$ standing for generators of the natural subgroups $S_{m}$ and $S_{n}$ fixing a point moved by $t$, respectively, and let $[M, N]$ denote the set of all commutators $[u, v]$ with $u \in M$ and $v \in N$. Then $\{A, B, t \mid \mathcal{R}, \mathcal{S},[M, N]\}$ is a presentation for $S_{m+n}$, again with the generator $t$ standing for a transposition. If $y$ and $z$ stand for an $(m+1)$-cycle in $S_{m+1}$ and an $(n+1)$-cycle in $S_{n+1}$, respectively, then zyt stands for an $(m+n)$-cycle in $S_{m+n}$.

Proof. Observe that there is a natural homomorphism from the group $G$ with the given presentation onto $S_{m+n}$ under which the subgroups generated by $M$ and $N$ map to $\operatorname{Sym}(\{1, \ldots, m\})$ and $\operatorname{Sym}(\{m+1, \ldots, m+n\})$, respectively, and $t$ maps to the transposition $(m, m+1)$.

Choose $m$ generators $a_{1}, a_{2}, \ldots, a_{m}$ for $\langle M, t\rangle=\langle A, t\rangle \cong S_{m+1}$ that satisfy the Moore presentation for $S_{m+1}$, with $\left\langle a_{1}, \ldots, a_{m-1}\right\rangle=\langle M\rangle$ and $a_{m}=t$; similarly, choose $n$ generators $b_{1}, b_{2}, \ldots, b_{n}$ for $\langle N, t\rangle=\langle B, t\rangle \cong S_{n+1}$ that satisfy the Moore presentation for $S_{n+1}$, with $b_{1}=t$ and $\left\langle b_{2}, \ldots, b_{n}\right\rangle=\langle N\rangle$. Also define $a_{m+j-1}=b_{j}$ for $1<j \leqslant n$, and let $S=\left\{a_{1}, \ldots, a_{m+n-1}\right\}$, a set of involutory generators for $\langle M, t, N\rangle=\langle A, t, B\rangle=G$.

Now consider a Coxeter relation $\left(a_{i} a_{j}\right)^{m_{i j}}=1$. If $1 \leqslant i \leqslant j \leqslant m$, then this holds in $\langle M, t\rangle$, or if $m \leqslant i \leqslant j \leqslant m+n-1$, then it holds in $\langle N, t\rangle$, and otherwise, if $1 \leqslant i<m<j$, then it holds since $a_{i} \in M$ commutes with $a_{j} \in N$. Thus $G=\langle S\rangle \cong S_{m+n}$. Finally, if $y$ and $z$ are elements of $G$ standing for the $(m+1)$-cycle $(1,2, \ldots, m+1)$ and the $(n+1)$-cycle $(m, m+1, \ldots, m+n)$, respectively, then $z$ ty stands for the $(m+n)$-cycle $(1,2, \ldots, m+n)$.

Corollary 3.2. Let $P=\{A, t \mid \mathcal{R}\}$ be a presentation for $S_{m+1}$, such that the generator $t$ stands for a transposition, and let $a$ and $c$ be words on the set $A \cup\{t\}$ standing for generators of the natural subgroup $S_{m}$ fixing a point moved by $t$. Then $\left\{A, b, t \mid \mathcal{R}, b^{2},(b t)^{3},[a, b],[c, b]\right\}$ is a presentation for $S_{m+2}$, with the generators $b$ and $t$ standing for transpositions. This presentation has $|A|+2$ generators, $|\mathcal{R}|+4$ 
relations, and length $\ell(P)+O(1)$. An element standing for an $(m+2)$-cycle in $S_{m+2}$ can be expressed in the form $y b$, where $y$ stands for an $(m+1)$-cycle in $S_{m+1}$.

Proof. Take $n=2, B=N=\{b\}$ and $M=\{a, c\}$ in Lemma 3.1

We next recall two facts which we will use to construct a special presentation of length $O(\log p)$ for $S_{p+2}$, when $p$ is an odd prime.

Proposition 3.3 (Sunday [19]). Let $p$ be an odd prime. Then $\operatorname{PSL}(2, p)$ has presentation $\left\{a, c \mid a^{p}, a c a c a c^{-1},\left(a^{(p+1) / 2} c a^{4} c\right)^{2}\right\}$, of length $O(\log p)$, where the generators $a$ and $c$ may be taken as standing, respectively, for the linear fractional transformations $z \mapsto z+1$ and $z \mapsto-1 / z$.

Proposition 3.4 (Burnside [6] and Miller [13). If $n \geqslant 3$, then $S_{n+1}$ has presentation $\left\{a_{1}, a_{2}, \ldots, a_{n} \mid a_{i}^{2},\left(a_{i} a_{j}\right)^{3},\left(a_{i} a_{j} a_{i} a_{k}\right)^{2}\right.$ for all distinct $\left.i, j, k\right\}$, with generator $a_{i}$ standing for the transposition $(i, n+1)$ for $1 \leqslant i \leqslant n$.

In the latter, it suffices to use for each unordered triple $\{i, j, k\}$ just one relation of the form $\left(a_{i} a_{j} a_{i} a_{k}\right)^{2}$, because the relator $\left(a_{i} a_{j} a_{i} a_{k}\right)^{2}$ is conjugate to $\left(a_{i} a_{k} a_{i} a_{j}\right)^{2}$ and equivalent to $\left(a_{j} a_{i} a_{j} a_{k}\right)^{2}$ since $\left(a_{i} a_{j}\right)^{3}=1$.

Theorem 3.5. Let $p$ be an odd prime, and let $\lambda$ be a primitive element of $\operatorname{GF}(p)$, with inverse $\mu$. Then

$$
\begin{aligned}
\{a, c, t \quad \mid & a^{p}, \operatorname{acacac}^{-1},\left(a^{(p+1) / 2} c a^{4} c\right)^{2}, \\
& \left.t^{2},[t, a],\left[t, c a^{\lambda} c a^{\mu} c\right],[t, c]^{3},\left(t t^{c} t t^{c a}\right)^{2},\left(t t^{c} t t^{c a^{\lambda}}\right)^{2},\left(a t^{c}\right)^{p+1}\right\}
\end{aligned}
$$

is a 3-generator 10-relator presentation of length $O(\log p)$ for $S_{p+2}$, in which att ${ }^{c}$ stands for a $(p+2)$-cycle and $t$ stands for a transposition. If $p \equiv 3 \bmod 4$, then the relator $\left(t t^{c} t t^{c a^{\lambda}}\right)^{2}$ is redundant and just 9 relators are needed.

Proof. Let $G$ be the group with the given presentation. Then there is an epimorphism $\theta: G \rightarrow S_{p+2}$, where $S_{p+2}$ acts on $\{\star, \infty\} \cup \mathrm{GF}(p)$, such that $\theta$ maps $t$ to the transposition $(\star, \infty)$, and maps $a$ and $c$ to elements that fix $\star$ and act on the projective line $\{\infty\} \cup \mathrm{GF}(p)$ in the same way as the two transformations given in Proposition 3.3. In particular, $a$ and $c$ generate a subgroup $H$ isomorphic to $\operatorname{PSL}(2, p)$, and the images of $a$ and $c a^{\lambda} c a^{\mu} c$ generate the stabiliser in $H$ of the points $\star$ and $\infty$ (and centralise $t$ ). Hence $t$ has exactly $p+1$ conjugates under the action of $H$, say $u_{i}$ for $i \in\{\infty\} \cup \operatorname{GF}(p)$, where $t=u_{\infty}$, and $\theta$ takes $u_{i}$ to the transposition $(\star, i)$ for each $i$.

Now the relations $t^{2}=[t, c]^{3}=\left(t t^{c} t t^{c a}\right)^{2}=\left(t t^{c} t t^{c a^{\lambda}}\right)^{2}=1$ give $u_{\infty}^{2}=\left(u_{\infty} u_{0}\right)^{3}=$ $\left(u_{\infty} u_{0} u_{\infty} u_{1}\right)^{2}=\left(u_{\infty} u_{0} u_{\infty} u_{\lambda}\right)^{2}=1$. Next, we use the facts that $\operatorname{PSL}(2, p)$ is doubly-transitive on the projective line $L=\{\infty\} \cup \mathrm{GF}(p)$, and has just two orbits on ordered triples of distinct points of $L$, namely the orbits of $(\infty, 0,1)$ and $(\infty, 0, \lambda)$. These give all that is required to invoke Proposition 3.4, and so the subgroup $K$ generated by these $p+1$ conjugates of $t$ is isomorphic to $S_{p+2}$. Moreover, if $p \equiv 3$ $\bmod 4$, then the relator $\left(t t^{c} t t^{c a^{\lambda}}\right)^{2}$ can be dropped because in that case $\operatorname{PSL}(2, p)$ has a single orbit on unordered triples of points of $L$ - see the observation after Proposition 3.4

On the other hand, the relator $\left(a t^{c}\right)^{p+1}$ may be written as $a^{p+1}$ times a product of conjugates of $t^{c}$, and as $a^{p}=1$ it follows that $a^{-1}$ is expressible as a product of conjugates of $t^{c}$ (and hence of $t$ ). In particular, $a$ lies in $K$, so all conjugates of $a$ lie 
in $K$, and as these conjugates generate $H \cong \operatorname{PSL}(2, p)$, it follows that $K$ contains $\langle a\rangle^{H}=H$, and thus $G=H K=K \cong S_{p+2}$.

We remark that, analogously, a 3-generator 6-relator presentation of length $O(\log p)$ due to Todd [21] for $\operatorname{PGL}(2, p)$ can be used to obtain a 4-generator 12relator presentation of length $O(\log p)$ for $S_{p+2}$.

Sass [17] used a presentation of Frasch [9] for $\operatorname{PSL}(2, p)$ to construct a presentation for $A_{p+2}$ with 3 generators, at most 9 relations, and length $O(\log p)$.

Ideally, we would like to convert one of our bounded short presentations for $S_{p+2}$ to one on its standard generators. Taking these to be $x=(\star, \infty)$ and $y=$ $(\star, \infty, 0,1, \ldots, p-1)$, and $a, c$ and $t$ as in Theorem 3.5. we have $x=t$ and $y=a t t^{c}$, and conversely, $a=y x y^{-1} x y$. We cannot, however, express $c$ as a short word in $x$ and $y$, and we suspect that this cannot be done.

Instead we apply Lemma 3.1 to Theorem 3.5 and obtain the following:

Theorem 3.6. Let $p$ and $q$ be odd primes, and let $\lambda$ and $\mu$ be primitive elements of $\operatorname{GF}(p)$ and $\operatorname{GF}(q)$, with inverses $\rho$ and $\sigma$, respectively. Then

$$
\begin{aligned}
\{a, c, d, f, t \quad \mid \quad & a^{p}, a c a c a c^{-1},\left(a^{(p+1) / 2} c a^{4} c\right)^{2}, d^{q}, d f d f d f^{-1},\left(d^{(q+1) / 2} f d^{4} f\right)^{2}, \\
& t^{2},[t, a],\left[t, c a^{\lambda} c a^{\rho} c\right],[t, d],\left[t, f d^{\mu} f d^{\sigma} f\right],[t, c]^{3},[t, f]^{3}, \\
& \left(t t^{c} t t^{c a}\right)^{2},\left(t t^{f} t t^{f d}\right)^{2},\left(t t^{c} t t^{c a^{\lambda}}\right)^{2},\left(t t^{f} t t^{f d^{\mu}}\right)^{2},\left(a t^{c}\right)^{p+1},\left(d t^{f}\right)^{q+1}, \\
& {\left.[a, d],[a, f],[c, d],[c f, t]^{2}\right\} }
\end{aligned}
$$

is a 5-generator 23-relator presentation of length $O(\log p+\log q)$ for $S_{p+q+2}$, in which $a t^{f} t t^{c} d$ stands for $a(p+q+2)$-cycle and $t$ stands for a transposition. If $p \equiv 3$ $\bmod 4$, then the relator $\left(t t^{c} t t^{c a^{\lambda}}\right)^{2}$ is redundant, and if $q \equiv 3 \bmod 4$, then the relator $\left(t t^{f} t t^{f d^{\mu}}\right)^{2}$ is redundant.

Proof. Take $m=p+1$ and $n=q+1$, and $A=\{a, c\}$ and $B=\{d, f\}$, with $M=\left\{a, t^{c t}\right\}$ and $N=\left\{d, t^{f t}\right\}$. Then using Lemma 3.1, we obtain the desired presentation for $S_{p+q+2}$, but with the relators $\left[a, t^{f t}\right],\left[d, t^{c t}\right]$ and $\left[t^{c t}, t^{f t}\right]$ in place of $[a, f],[c, d]$ and $[c f, t]^{2}$. Since $t$ commutes with both $a$ and $d$ in this group, the relations $[a, f]=1$ and $[c, d]=1$ imply that $\left[a, t^{f t}\right]=1$ and $\left[d, t^{c t}\right]=1$; further the relation $\left[t^{c t}, t^{f t}\right]=1$ is equivalent to $1=\left[t^{c}, t^{f}\right]=(\text { ctcftf })^{2}$ and hence to $[c f, t]^{2}=(f c t c f t)^{2}=1$.

Proof of Theorem 1.2. Suppose $n$ is even. If $n-2$ is the sum of two odd primes (as implied by the Goldbach conjecture), then Theorem 3.6 gives a bounded presentation of length $O(\log n)$ for $S_{n}$, in which the $n$-cycle $(1,2, \ldots, n)$ can be expressed as a word of length 9 in the generators. Adding an additional generator to represent this $n$-cycle then gives a special presentation with the required properties. Similarly, if $n$ is odd and $n-3$ is the sum of two odd primes, then the above argument gives a suitable presentation for $S_{n-1}$, to which Corollary 3.2 may be applied.

Even if the Goldbach conjecture is false, for sufficiently large $n$ a theorem of Vinogradov 22] implies that $n-2$ or $n-3$ is expressible as a sum of three odd primes $p, q, r$. Theorem 3.6 then gives a 5-generator 23-relator presentation for $S_{q+r+2}$, which can be used with Theorem 3.5 and Lemma 3.1 to produce a presentation for $S_{p+q+r+2}=S_{n}$ or $S_{n-1}$, with $5+3-1=7$ generators and at most $23+10+2 \times 2=37$ relations, and length $O(\log n)$. The appropriate long cycle is expressible as a word of length $9+1+5=15$ in the generators, and the rest follows easily. 
Alternatively, we can resort to a theorem of Ramaré [16] which states that every even positive integer is a sum of at most six primes. We partition the primes into three pairs, use Theorem 3.5] or Theorem 3.6 as appropriate for each pair, and then apply Lemma 3.1 twice to glue the three presentations together. For even $n$, this gives a presentation for $S_{n}$ on at most 13 generators with at most $(23+23+4)+23+4=77$ relations, with the $n$-cycle $(1,2, \ldots, n)$ expressible as a word of length at most $(9+1+9)+1+9=29$ in the generators. For odd $n$, again we can construct a suitable presentation for $S_{n-1}$, and then apply Corollary 3.2

\section{Alternating Groups}

Presentations for the alternating groups $A_{n}$ are obtainable easily from those for $S_{n}$, using the Reidemeister-Schreier process (as described in [18, Chapter 6]).

If $P$ is any presentation for $S_{n}$, let $E$ and $O$ be the subsets of generators in $P$ that stand for even and odd permutations in $S_{n}$, respectively. For a Schreier transversal for $A_{n}$ in $S_{n}$, we may take $\{1, t\}$ where $t$ is any one of the generators in $O$ (such as one standing for a single transposition). The Schreier generators for $A_{n}$ then consist of all elements of the form $u$ and $t u t^{-1}$ where $u \in E$, together with all elements of the form $t v^{ \pm 1}$ where $v \in O$, and the Reidemeister-Schreier relators are just those in the presentation for $S_{n}$, rewritten in terms of these Schreier generators, plus conjugates of these by the element $t^{-1}$.

Hence if $Q$ is the resulting presentation for $A_{n}$, then $d(Q) \leqslant 2 d(P)-2, r(Q) \leqslant$ $2 r(P)$ and $\ell(Q) \leqslant 2 \ell(P)$.

If $P$ is defined on the standard generators $x=(1,2)$ and $y=(1,2, \ldots, n)$, then the presentation $Q$ for $A_{n}$ is on the two $n$-cycles $y$ and $x y x$ when $n$ is odd, or the two $(n-1)$-cycles $x y$ and $x y^{-1}$ when $n$ is even. Note also that if $P$ is a special presentation with a bounded number of generators and relations, then any nonstandard generator can be eliminated by the addition of one further relation, and so $P$ gives rise to a presentation for $S_{n}$ on the standard generators, and hence to a 2-generator presentation for $A_{n}$, with a bounded number of relations.

Thus we obtain Theorem 1.3 as a consequence of Theorems 1.1 and 1.2 .

\section{REFERENCES}

1. L. Babai, A.J. Goodman, W.M. Kantor, E.M. Luks, and P.P. Pálfy, Short presentations for finite groups. J. Algebra 194 (1997), 79-112. MR.1461483 (98h:20044)

2. L. Babai, W.M. Kantor and A. Lubotzky, Small-diameter Cayley graphs for finite simple groups. European J. Combin. 10 (1989), no. 6, 507-522. MR1022771 (91a:20038)

3. László Babai and Endre Szemerédi, On the complexity of matrix group problems, I. In Proc. 25th IEEE Sympos. Foundations Comp. Sci., pages 229-240, 1984.

4. László Babai, Randomization in group algorithms: conceptual questions. Groups and Computation, II (New Brunswick, NJ, 1995), 1-17, DIMACS Ser. Discrete Math. Theoret. Comput. Sci., 28, Amer. Math. Soc., Providence, RI, 1997. MR1444127|(98k:68092)

5. Robert Beals, Charles R. Leedham-Green, Alice C. Niemeyer, Cheryl E. Praeger, and Ákos Seress, A black-box group algorithm for recognizing finite symmetric and alternating groups. I. Trans. Amer. Math. Soc. 355 (2003), 2097-2113. MR1953539(2003k:20002)

6. W. Burnside, Theory of Groups of Finite Order, 2nd ed., Dover Publications, Inc., New York, 1955, xxiv+512 pp. MR0069818 (16:1086c)

7. C.M. Campbell, G. Havas, C. Ramsay, and E.F. Robertson, Nice efficient presentations for all small simple groups and their covers. London Math. Soc. J. Comput. Math. 7 (2004), 266-283. MR 2118175 (2006g:20046)

8. H.S.M. Coxeter and W.O.J. Moser, Generators and Relations for Discrete Groups, 4th ed., Springer-Verlag (Berlin), 1980, ix+169 pp. MR562913 (81a:20001) 
9. H. Frasch, Die Erzeugenden der Hauptkongruenzgruppen für Primzahlstufen. Math. Ann. 108 (1933), 229-252. MR 1512847

10. R.M. Guralnick, W.M. Kantor, M. Kassabov and A. Lubotzky, Presentations of finite simple groups: a quantitative approach. J. Amer. Math. Soc. 21 (2008), 711-774. MR2393425

11. R.M. Guralnick, W.M. Kantor, M. Kassabov and A. Lubotzky, Presentations of finite simple groups: a computational approach. To appear J. European Math. Soc.

12. Alexander Hulpke and Ákos Seress, Short presentations for three-dimensional unitary groups. J. Algebra, 245 (2001), 719-729. MR.1863898(2002m:20079)

13. G. A. Miller, Abstract definitions of all the substitution groups whose degrees do not exceed seven, Amer. J. Math. 33 (1911), 363-372. MR 1506130

14. E.H. Moore, Concerning the abstract groups of order $k$ ! and $\frac{1}{2} k !$, Proc. London Math. Soc. 28 (1897), 357-366.

15. E.A. O'Brien, Towards effective algorithms for linear groups. Finite Geometries, Groups and Computation, Colorado, September, 2004. De Gruyter, Berlin, 163-190, 2006. MR2258009 (2008c:20022)

16. O. Ramaré, On Šnirelman's constant. Ann. Scuola Norm. Sup. Pisa Cl. Sci. (4) 22 (1995), 645-706. MR.1375315 (97a:11167)

17. Hartmut Sass, Eine abstrakte Definition gewisser alternierender Gruppen. Math. Z. 128 (1972), 109-113. MR0323875 (48:2228)

18. Charles C. Sims, Computation with finitely presented groups. Cambridge University Press, 1994, xiii+604 pp. MR.1267733 (95f:20053)

19. J.G. Sunday, Presentations of the groups SL $(2, m)$ and PSL $(2, m)$. Canad. J. Math. 24 (1972), 1129-1131. MR0311782 (47:344)

20. Michio Suzuki, On a class of doubly transitive groups. Ann. of Math. (2) 75 (1962), 105-145. MR0136646 (25:112)

21. J.A. Todd, A note on the linear fractional group. J. London Math. Soc. 7 (1932), 195-200.

22. I.M. Vinogradov, The method of trigonometrical sums in the theory of numbers (Russian). Trav. Inst. Math. Stekloff 23 (1947), 109 pp. MR0029417(10:599a)

School of Mathematical Sciences, Queen Mary, University of London, London E1 4NS, UNITED KINGDOM

Department of Mathematics, University of Auckland, Auckland, New Zealand

School of Mathematical Sciences, Queen Mary, University of London, London E1 4NS, UNiTED Kingdom

Department of Mathematics, University of Auckland, Auckland, New Zealand 